\title{
Heightened Defensive Responses Following Subtotal Lesions of Macaque Orbitofrontal Cortex
}

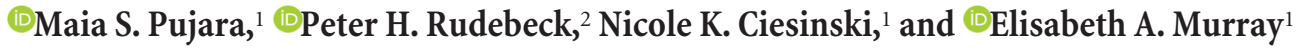 \\ ${ }^{1}$ Section on the Neurobiology of Learning and Memory, Laboratory of Neuropsychology, National Institute of Mental Health, National Institutes of Health, \\ Bethesda, Maryland 20892, and 2Friedman Brain Institute, Icahn School of Medicine at Mount Sinai, New York, New York 10029
}

Anxiety disorders are characterized by excessive attention to threat. Several brain areas, including the orbitofrontal cortex (OFC), have been associated with threat processing, with more recent work implicating specialized roles for the medial and lateral subregions of the OFC in mediating specific symptoms of anxiety disorders. Virtually no causal work, however, has evaluated the role of these OFC subregions in regulating behavioral responses under threat. To address this gap, we compared male rhesus monkeys (Macaca mulatta) with bilateral excitotoxic lesions restricted to either the lateral OFC (1OFC), targeting Walker's areas 11 and 13, or the medial OFC ( $\mathrm{mOFC}$ ), targeting Walker's area 14, to a group of unoperated controls on behavioral responses to the presentation of a fake rubber snake, fake spider, and neutral stimuli. Both lesion groups showed heightened defensive and reduced approach responses, accompanied by longer latencies to retrieve a food reward, in the presence of the threatening stimuli. Compared to unoperated controls, the mOFC lesion group also showed longer latencies to reach for rewards and a greater proportion of defensive responses (e.g., piloerection) in the presence of neutral stimuli. Thus, monkeys with mOFC lesions displayed a greater tendency to express defensive responses even in the absence of threat. Overall, our data reveal that both the $\mathrm{mOFC}$ and lOFC contribute to the attenuation of defensive responses. Notably, these findings, obtained following selective, excitotoxic lesions of the OFC, are diametrically opposed to the effects of aspiration lesions of OFC observed in macaques.

Key words: defense; emotion; fear; lesion; macaque; orbitofrontal

\section{Significance Statement}

Engaging in adaptive defensive responses under threat promotes biological fitness. The orbitofrontal cortex (OFC) has been implicated in regulating defensive responses to threat, with distinct subregions likely playing different roles. Here we tested the effects of excitotoxic damage restricted to either the lateral or medial subdivisions of the OFC in rhesus macaques. We found significantly heightened defense and reduced approach responses to threatening stimuli in both lesion groups. While lateral OFC lesions led to an increase in defense responses to the threatening stimuli, medial OFC lesions produced increases in defense responses to both threatening and neutral stimuli. Our findings provide insights into the neural regulation of defensive responses to threat and inform the etiology and treatment of anxiety disorders in humans.

\section{Introduction}

Engaging in adaptive defensive responses under threat promotes biological fitness. In nonhuman primates, such predators as

\footnotetext{
Received Oct. 30, 2018; revised March 4, 2019; accepted March 10, 2019.

Author contributions:P.H.R. and E.A.M. designed research;P.H.R., N.K.C., and E.A.M. performed research; M.S.P., P.H.R., and N.K.C. analyzed data; M.S.P. and E.A.M. wrote the paper.

This work was supported by the Intramural Research Program of the National Institute of Mental Health (E.A.M., ZIAMH002887). We thank Dawn Lundgren, Emily Moylan, Lily Chau, Anna Prescott, and Brittney Thompson for assistance with data collection; and Richard Saunders and Rachel Reoli for help performing surgery. We also thank the staff of the Nuclear Magnetic Resonance Facility, National Institute of Neurological Disorders and Stroke, and the Laboratory of Diagnostic and Radiology Research, as well as Megan Malloy for assistance in obtaining the MR scans. The authors declare no competing financial interests.

Correspondence should be addressed to Elisabeth A. Murray at murraye@mail.nih.gov.

https://doi.org/10.1523/JNEUROSCI.2812-18.2019

Copyright $\odot 2019$ the authors
}

snakes, either fake or real, provoke a number of canonical defensive behavioral responses, including freezing, eye and head aversion, and withdrawal (Meunier et al., 1999; Nelson et al., 2003). Repeated encounters with a threat stimulus in the absence of aversive outcomes typically leads to extinction of these defensive responses (Nelson et al., 2003; Izquierdo and Murray, 2004; Izquierdo et al., 2005; Chudasama et al., 2008), demonstrating adaptability in the face of threat versus safety. Maladaptive responses present as either under-responding to immediate threat, leading to vulnerability to predation, or over-responding, leading to a reduced ability to engage in essential activities, such as foraging. Because excessive attention or bias to threat characterizes individuals with anxiety disorders (Mogg and Bradley, 2018), identifying the brain areas involved in regulating responses to threat holds some clinical relevance. 
Neuroimaging studies in humans and causal manipulation studies in monkeys implicate similar sets of brain regions in the adaptive response to threat, including the amygdala, hippocampus, bed nucleus of the stria terminalis, and periaqueductal gray (PAG; Fox et al., 2010; DesJardin et al., 2013; Forcelli et al., 2016, 2017; LeDoux and Pine, 2016). Recent work also highlights a role for the orbitofrontal cortex (OFC; Shiba et al., 2016; Patrick et al., 2019). The OFC is a large, heterogeneous structure, and some studies in humans suggest functional specializations of IOFC and mOFC in mediating the relationship between emotional reactivity and symptoms of anxiety disorders. For example, a comparison of the neural responses of spider phobic and nonphobic subjects to spider stimuli revealed decreased activity in the lOFC, which was normalized by cognitive behavioral therapy treatment (Schienle et al., 2007). In another study, individuals with generalized anxiety disorder (GAD) exhibited, relative to controls, deficient recruitment of the mOFC during fear generalization (Greenberg et al., 2013). Recent lesion and stimulation studies in monkeys and humans, respectively, further reveal distinctions between OFC subregions in regulating responses to threat and mood (Shiba et al., 2014; Rao et al., 2018).

Differences in the anatomical connectivity of the lOFC versus the mOFC may underlie the observed differences in threat and affective processing (Price, 2005). The lOFC (areas 11/13) projects directly to the amygdala, whereas the mOFC (area 14) projects to midbrain structures, including the hypothalamus and PAG, in addition to the amygdala (Carmichael and Price, 1995a,b). Whereas the amygdala is thought to regulate responses specifically in the context of a predator-specific threat (Martinez et al., 2011; Bindi et al., 2018), electrical, pharmacological, and optogenetic stimulation of the PAG has been shown to produce defensive behaviors even in the absence of a threat stimulus (Motta et al., 2017). Area 11/13 may therefore contribute to regulating defensive responses in the context of perceived threat only, whereas area 14 may play a more general role in emotional reactivity. Although subregional distinctions have been made with respect to the OFC and reward-guided learning and decision making (Noonan et al., 2010b; Rudebeck and Murray, 2011; Murray et al., 2015; Murray and Rudebeck, 2018), the individual contributions of OFC subregions to behavioral regulation under threat in rhesus macaques remain less well understood.

To explore the contributions of OFC subregions in marshaling defensive responses under threat, we compared groups of rhesus monkeys (Macaca mulatta) that received bilateral, excitotoxic lesions of either the lOFC or the mOFC to a group of unoperated controls. All monkeys were assessed for their behavioral responses to threat, as indexed by (1) their latency to reach for a food reward and (2) observed approach and defense responses during repeated presentations of a fake rubber snake and spider. We expected both experimental groups to display heightened defensive responses, with IOFC damage producing specific defensive responses to the threatening stimuli and mOFC damage producing nonspecific heightened defensive responses to both threatening and neutral stimuli.

\section{Materials and Methods}

Subjects. Twenty adult rhesus monkeys (Macaca mulatta), three female, served as subjects. Four male monkeys sustained bilateral excitotoxic lesions of the lOFC (targeting areas 11 and 13), and four male monkeys sustained bilateral excitotoxic lesions of the mOFC (targeting area 14). Data from the same operated monkeys on tests of learning and decision making have been previously reported (Rudebeck and Murray, 2011). The remaining 12 monkeys (nine males) were retained as unoperated controls. Four were trained concurrently with the two operated groups (Rudebeck and Murray, 2011), and the remaining eight were historical controls studied by Rudebeck et al. (2013a). The training histories of all the monkeys were highly similar. All monkeys had received training on tests of learning and decision making before beginning the experiments described here. No statistical test was run to determine the sample size $a$ priori. The sample sizes we chose are similar to those used in previous publications. Monkeys weighed between 4.6 and $9.7 \mathrm{~kg}$ and all were $\geq 4$ years old at the start of testing. Each animal was individually or pairhoused, kept on a 12-h light/dark cycle (lights on at 7:00 A.M.), maintained on primate chow and fruit, and given access to water $24 \mathrm{~h}$ a day. All procedures were reviewed and approved by the National Institute of Mental Health Animal Care and Use Committee.

Surgery. Eight monkeys received injections of the neurotoxin ibotenic acid, which targeted either the lOFC (Walker's areas 11 and 13) or the mOFC (Walker's area 14) bilaterally (Rudebeck and Murray, 2011; Walker, 1940). For the purpose of relating the location of our intended lesions to other commonly used anatomical frameworks, we note that the $1 \mathrm{OFC}$ corresponds approximately to areas $131,13 \mathrm{~m}, 13 \mathrm{~b}, 11 \mathrm{l}$, and $11 \mathrm{~m}$, and the mOFC corresponds approximately to areas $14 \mathrm{r}, 14 \mathrm{c}$, and $10 \mathrm{~m}$ of Carmichael and Price (1994). Monkeys were given $\geq 2$ weeks to recover from surgery before postoperative behavioral testing was initiated.

Lesion assessment. Lesions were assessed using T2-weighted MRI scans obtained within 1 week of the surgery. The location and extent of excitotoxic lesions in the cortex is reliably indicated by the white hypersignal on T2-weighted scans (Málková et al., 2001; c.f. Basile et al., 2017). The details of the lesion assessment method are described by Rudebeck and Murray (2011). Briefly, MR scan slices were matched to drawings of coronal sections from a standard rhesus monkey brain at 1-mm intervals. We then plotted the extent of the hypersignal onto standard sections and estimated the volume of the lesion in each operated monkey from those plots. The estimated extent and location of the lesions are shown in Figure $1 A$ for the $1 \mathrm{OFC}$ and Figure $1 B$ for the mOFC. Mean percentage of estimated damage from the T2-weighted MRI scans for intended and unintended (i.e., hypersignal that extended beyond the boundaries of the intended lesion) regions for each group is provided in Table 1.

Apparatus and materials. Monkeys were trained in a modified Wisconsin General Test Apparatus (WGTA) located in a darkened room. A clear Plexiglas box measuring $11.4 \mathrm{~cm}$ (width) $\times 71.1 \mathrm{~cm}$ (length) $\times 11.4 \mathrm{~cm}$ (height) was placed within the test compartment of the WGTA. The box was hinged at the back, which enabled the experimenter to easily lift the back flap and place objects within the box in between trials. Test objects included the following: a gray/green rubber snake measuring $50.8 \mathrm{~cm}$ in length and $\sim 2 \mathrm{~cm}$ in diameter, a black, "hairy" rubber spider measuring $10 \mathrm{~cm}$ in width $\times 13.5 \mathrm{~cm}$ in length $\times 2.5 \mathrm{~cm}$ in height, and eight neutral junk objects varying in size, shape, and color. Another three junk objects were dedicated to pretraining. Food rewards consisted of fruit snacks (Giant Food).

Each monkey's behavior during each session was recorded on videotape. The video-recording setup consisted of the following: two video cassette recorders (VCRs, SR-TS1U), two color video monitors (TMH1375SU), and two video CCTV cameras (TK-CJ20U), all from JVC Professional Product Company. The VCRs were equipped with Society for Motion Picture and Television Engineers time-code generators (Horita, TG-50), which were synchronized at the beginning of each session. The dual-camera setup enabled the test sessions to be videotaped from two vantage points: Camera 1 provided a view of the test compartment from above, and Camera 2 provided a frontal view of the monkeys' behavioral reactions to the objects in the Plexiglas box.

Experimental design and statistical analysis. All monkeys were evaluated for their behavioral responses to objects known to be threatening (snake, spider) or nonthreatening (neutral objects) to macaques. In addition, before coming to the present study, all monkeys had experience in the test apparatus used here, as well as a general familiarity with approaching and displacing small junk objects (see Subjects).

Pretraining. All monkeys were first required to retrieve food rewards located on top of the Plexiglas box while the box was empty for 20 trials. For another 10 trials, one of the three pretraining objects was placed inside the box. This was repeated for one more day or until the monkey 
A

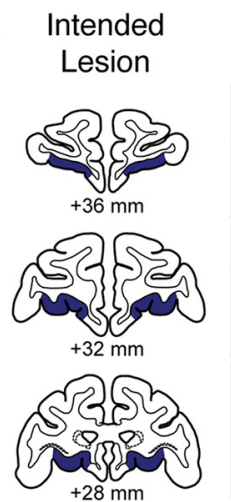

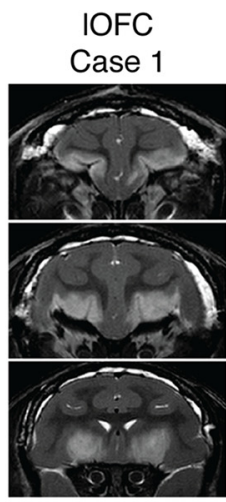

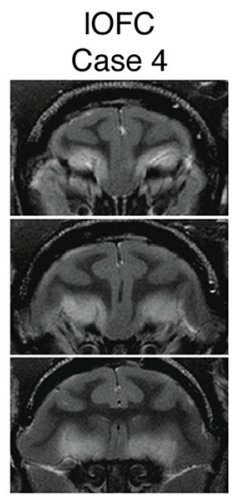

B

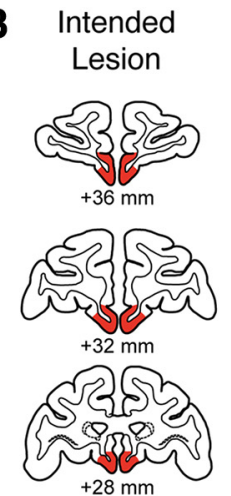

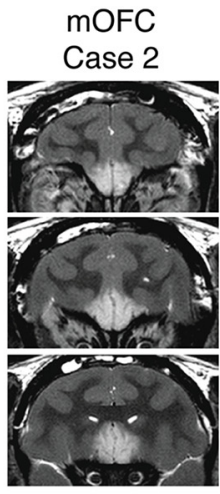

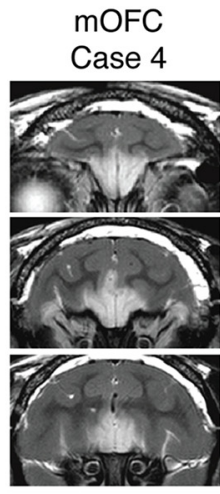

Figure 1. $\quad \boldsymbol{A}, \boldsymbol{B}$, Excitotoxic lesions of IOFC area $11 / 13(\boldsymbol{A})$ and $\mathrm{mOFC}$ area $14(\boldsymbol{B})$. The left column in each panel shows the extent of the intended lesion area (blue, IOFC; red, $\mathrm{m} 0 \mathrm{FC})$ on standard coronal sections through the frontal lobe of a macaque brain $36-28 \mathrm{~mm}$ rostral to the interaural plane. The middle and right columns in each panel show coronal images at corresponding levels taken from T2-weighted MRI scans obtained within 1 week of surgery from Cases 1 and 4 from the IOFC group and Cases 2 and 4 from the m0FC group. White hypersignal is associated with edema that follows injections of the excitotoxin ibotenic acid and typically corresponds to the extent of the lesion.

Table 1. Estimated percentage damage to $10 \mathrm{FC}$ and $\mathrm{mOFC}$

\begin{tabular}{|c|c|c|c|c|c|c|}
\hline \multirow[b]{2}{*}{ Monkey } & \multicolumn{3}{|l|}{ IOFC } & \multicolumn{3}{|l|}{$\mathrm{mOFC}$} \\
\hline & $\begin{array}{l}\text { Left } \\
\text { hemisphere }\end{array}$ & $\begin{array}{l}\text { Right } \\
\text { hemisphere }\end{array}$ & Mean ${ }^{c}$ & $\begin{array}{l}\text { Left } \\
\text { hemisphere }\end{array}$ & $\begin{array}{l}\text { Right } \\
\text { hemisphere }\end{array}$ & Mean' \\
\hline \multicolumn{7}{|c|}{$\mathrm{IOFC}_{\text {group }}{ }^{a}$} \\
\hline Case 1 & 80.8 & 82.7 & 81.7 & 7.5 & 4.1 & 5.8 \\
\hline Case 2 & 89.7 & 93.8 & 91.8 & 3.8 & 3.0 & 3.4 \\
\hline Case 3 & 79.4 & 86.7 & 83.1 & 1.7 & 3.0 & 2.3 \\
\hline Case 4 & 89.6 & 84.1 & 86.8 & 3.9 & 5.2 & 4.6 \\
\hline Mean & & & 85.9 & & & 4.0 \\
\hline \multicolumn{7}{|c|}{$\mathrm{m0FC}$ group ${ }^{b}$} \\
\hline Case 1 & 12.7 & 17.0 & 14.9 & 98.6 & 93.4 & 96.0 \\
\hline Case 2 & 7.7 & 8.2 & 8.0 & 77.8 & 76.5 & 77.1 \\
\hline Case 3 & 29.3 & 3.6 & 16.4 & 42.3 & 75.2 & 58.7 \\
\hline Case 4 & 13.2 & 4.1 & 8.7 & 90.6 & 79.8 & 85.2 \\
\hline Mean & & & 12.0 & & & 79.3 \\
\hline
\end{tabular}

${ }^{a}$ Monkeys with injections of ibotenic acid targeting areas 11 and 13 .

${ }^{b}$ Monkeys with injections of ibotenic acid targeting area 14.

'Average of the damage to left and right hemispheres.

readily retrieved a food reward from the top, back edge of the Plexiglas box with and without an object present. Thus, by the end of pretraining, monkeys were familiar with the Plexiglas box, and with the procedure of reaching for rewards in the presence of an object.

Main task. Monkeys performed five sessions composed of 10 trials each. A different object was used for each trial within a session, and no new objects were introduced from session to session. In eight trials, one of the neutral objects was placed on the surface of the test compartment in the center of the Plexiglas box. In the remaining two trials, the rubber snake or spider was placed in the center of the Plexiglas box. The snake and spider objects were static and presented pseudorandomly in the sequence of 10 trials with the constraint that neither the snake nor spider was presented on the first trial of the session. Trials were separated by 20 s. Sessions were administered every other day.

On each trial, the opaque screen that separated the monkey from the test compartment was raised. The monkey was free to reach over the object to retrieve the food reward. Each trial lasted $30 \mathrm{~s}$ regardless of whether the food was retrieved. At the end of the trial, the opaque screen separating the monkey from the test compartment was lowered.

Videotape analysis. Food-retrieval latencies were derived from analysis of the video recordings from Camera 1, which provided a top-down view of the compartment. Latencies were scored to the nearest frame and had a resolution of $\sim 4 \mathrm{~ms}$. Time for the latency measure was initiated when the opaque screen was raised $\sim 15 \mathrm{~cm}$ above the test tray. This could be discerned in the videotape by a mark on the cage, visible in the view of Camera 2, which provided a frontal view of the monkeys' reactions to the objects in the Plexiglas box. The response was considered complete when
Table 2. Description of behaviors scored

\begin{tabular}{lll}
\hline Category & Behavior & Description \\
\hline Approach & Move forward & Shifts body forward, closer to stimulus \\
& Touch & Handles Plexiglas box with hand or foot \\
& Take/eat reward & Picks up or mouths the food reward \\
Defense & Freezing & Motionless for $\geq 3 \mathrm{~s}$ \\
& Startle & Jerks suddenly \\
& Eye/head aversion & Avoids eye contact or turns head away for $\geq 3 \mathrm{~s}$ \\
& Piloerection & Hair stands on end \\
& Move away/withdrawal & Retreats to back of the cage, away from stimulus, \\
& & standing with a tense posture \\
\hline Definitions for each behavior scored within approach and defense behavior categories.
\end{tabular}

the monkey grasped the food reward just before it withdrew its arm. If no response was made within the trial limit of $30 \mathrm{~s}$, a score of $30 \mathrm{~s}$ was recorded.

Behaviors expressed during each trial were scored from video recordings from Camera 2. Two main categories were observed: approach and defense. Table 2 provides a list and definitions of the behaviors that comprised the approach and defense categories. Approach and defense behaviors are standard measures in this and other laboratories (Meunier et al., 1999; Izquierdo and Murray, 2004; Kalin et al., 2004; Machado and Bachevalier, 2008; Chudasama et al., 2009; Machado et al., 2009). Approach was scored if the monkey moved from the back to the front of the cage and stayed close to the Plexiglas box, obtained the reward, or touched the Plexiglas box in which the object was contained. Defense was scored when the monkey froze, showed piloerection, averted his/her eyes, shifted his/her whole head to avoid eye contact with the object, or moved away from the object and/or stayed in the back of the cage (withdrawal). We combined the eye and head aversion categories for the final analyses, given that the occurrences of these behaviors highly overlapped. The front-facing (Camera 2) video recording for the fourth session of a monkey (Case 2) in the lOFC group was missing. We therefore analyzed data from only four sessions for this monkey, instead of five.

Behaviors were scored by an observer naïve to group assignment. Each behavior listed in Table 2 was scored with either a " 0 " if it was absent in each trial, or a " 1 " if it was present in each trial. It was possible for an approach behavior and defense behavior to have been present and scored simultaneously within the same trial. For example, the observer may have scored a "take/eat reward" behavior as a 1 if the monkey took the reward, in addition to scoring "move away/withdrawal" as a 1 if the monkey also moved away or withdrew from the stimulus either before or after taking the food. A subset of sessions was subsequently scored independently by a second observer who was aware of the group assignments. Interobserver reliability was obtained by calculating a Pearson's correlation coefficient for the total proportion of behaviors averaged across all trials and all five sessions per monkey, for a sample of eight monkeys total (two 

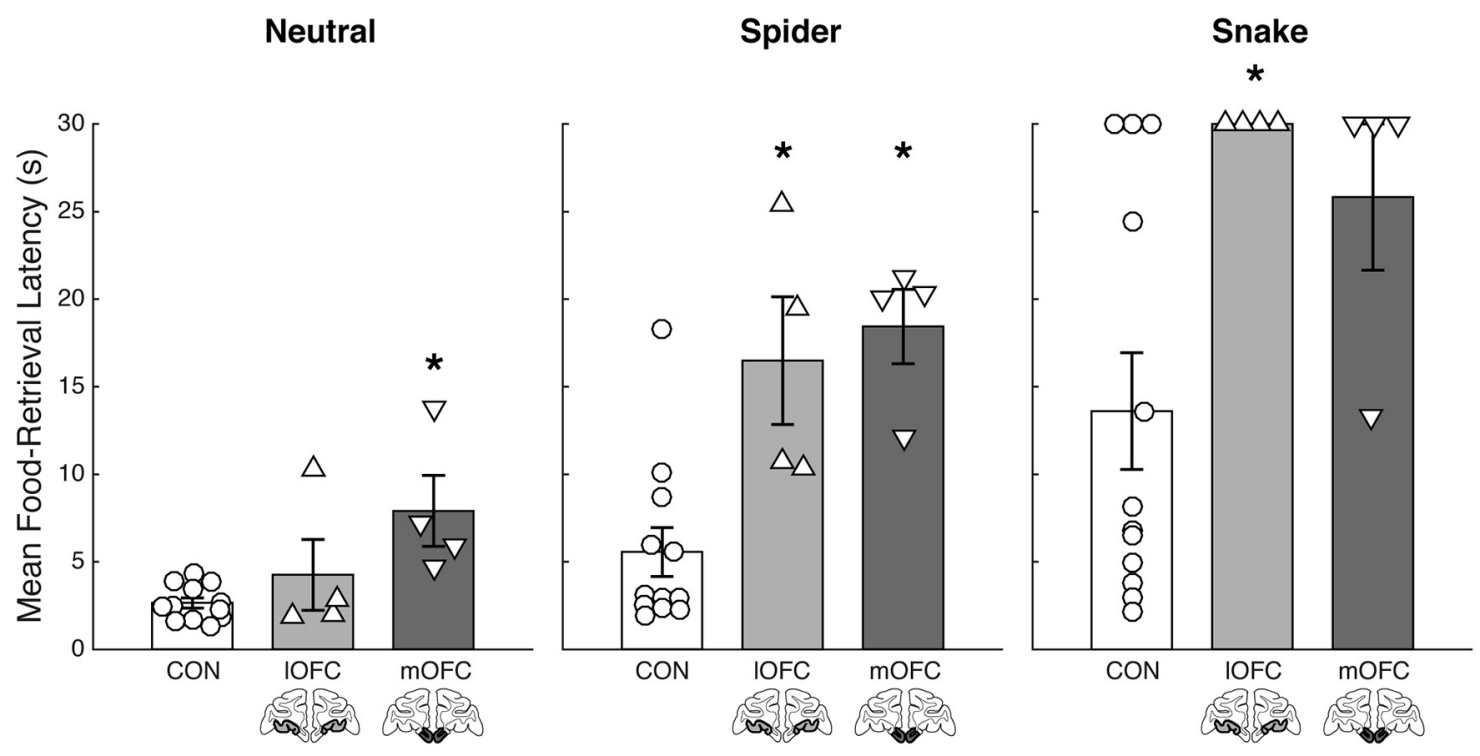

Figure 2. Average food-retrieval latencies collapsed across all five sessions are plotted for each group for the neutral, spider, and snake trial types separately. The IOFC lesion group, is represented in light gray on a standard coronal section below each corresponding bar. The $\mathrm{mOFC}$ lesion group, is represented in dark gray on a standard coronal section below each corresponding bar. Unoperated controls (CON) are represented in white. Error bar equals SEM; ${ }^{*} p<0.05$ for a comparison of each subtotal OFC lesion group compared with the control group.

monkeys from each of the experimental groups and four unoperated control monkeys).

Statistical analysis. Because of the small sizes of our experimental groups, we tested for normality of our data and found that our data violated assumptions of normality. This was true regardless of the type of transformation used to normalize the distributions (log, square root, cube root transformation, Tukey's ladder of power; all Shapiro-Wilk tests, $p<0.05)$. We therefore used nonparametric tests for all of our analyses.

To test the effects of subtotal OFC lesions on responses to the stimuli, we ran a separate two-tailed Kruskal-Wallis test for each stimulus category, followed by post hoc Mann-Whitney $U$ tests to compare the experimental lesion groups to the intact control group and to each other. To determine whether animals habituated to the stimuli over repeated presentations, we next performed within-group comparisons of foodretrieval latencies between the first and fifth sessions (using the Wilcoxon signed-rank test for paired data), as well as between-group comparisons of latencies in the fifth session (Mann-Whitney $U$ test).

To test whether whole-body responses corroborated the effects observed from the food-retrieval latency scores, we determined the proportion of trials with approach responses and proportion of trials with defense responses. We then calculated an approach-defense index (ADI) by computing the difference between the proportion of trials with approach (APP) versus defense (DEF) behaviors and then normalizing the difference by the sum (Eq. 1):

$$
A D I=\frac{P_{A P P}-P_{D E F}}{P_{A P P}+P_{D E F}}
$$

We ran separate two-tailed Kruskal-Wallis tests for each stimulus category, followed by post hoc Mann-Whitney $U$ tests for between-group comparisons. Behavioral data were collapsed across all sessions for each stimulus category.

\section{Results}

\section{Food-retrieval latencies}

To establish the effect of $1 \mathrm{OFC}$ and $\mathrm{mOFC}$ lesions on responses to the threatening stimuli, we first analyzed food-retrieval latencies. For our initial analysis, the data for all three stimulus categories were averaged across session, and the main effect of group was determined for each category (Fig. 2). There was an overall significant effect of group on food-retrieval latency for the spider $\left(\chi^{2}=12.23, p=0.002\right)$ and snake $\left(\chi^{2}=7.80, p=0.02\right)$ stimuli. Between-group comparisons indicated that both operated groups took longer to reach for the food reward in the presence of both the spider (lOFC: $U=2, p=0.004$; mOFC: $U=1, p=$ 0.002 ) and the snake (lOFC: $U=6, p=0.03$; mOFC: $U=8.5, p=$ 0.06 , approaching significance) compared with controls, whereas the operated groups did not differ from each other (spider: $U=5$, $p=0.49$; snake: $U=10, p=0.45$ ). There was also a significant effect of group on food-retrieval latency for the neutral stimuli $\left(\chi^{2}=7.88, p=0.02\right)$, which was driven by a difference between the mOFC lesion group and controls $(U=0, p=0.001)$, but not the lOFC lesion group and controls $(U=20, p=0.68)$. Thus, the mOFC lesion group showed significantly longer food-retrieval latencies compared to controls in the presence of the neutral stimuli. To isolate these effects, we subtracted the mean foodretrieval latency for trials with neutral objects from the mean for each threat stimulus and found that the mOFC group did not differ from either the lOFC (spider vs neutral: $U=8, p=1.00$; snake vs neutral: $U=13, p=0.20$ ) or controls (spider vs neutral: $U=13, p=0.21$; snake vs neutral: $U=21, p=0.77$ ). The lOFC group, however, did differ from controls (spider vs neutral: $U=$ $2, p=0.004$; snake vs neutral: $U=4, p=0.01$ ), which shows that any differences between the experimental groups are due to a difference in how the mOFC group responded to the neutral stimuli.

The long food-retrieval latencies displayed by the mOFC lesion group on trials with neutral stimuli raised a question about whether the increased latency in the presence of the neutral stimuli might be a by-product of a prolonged affective reaction following exposure to the fake snake. To determine whether the heightened food-retrieval latency effects for the mOFC group were more pronounced on neutral trials immediately following the snake trial, we performed two Kruskal-Wallis tests on the food-retrieval latencies for neutral trials immediately before and after the snake trial. If there was an influence of the snake presentation on food-retrieval latencies for the mOFC group, we would have expected to see a significant effect of group for the trials after, but not before, the snake. Instead, however, we found a 

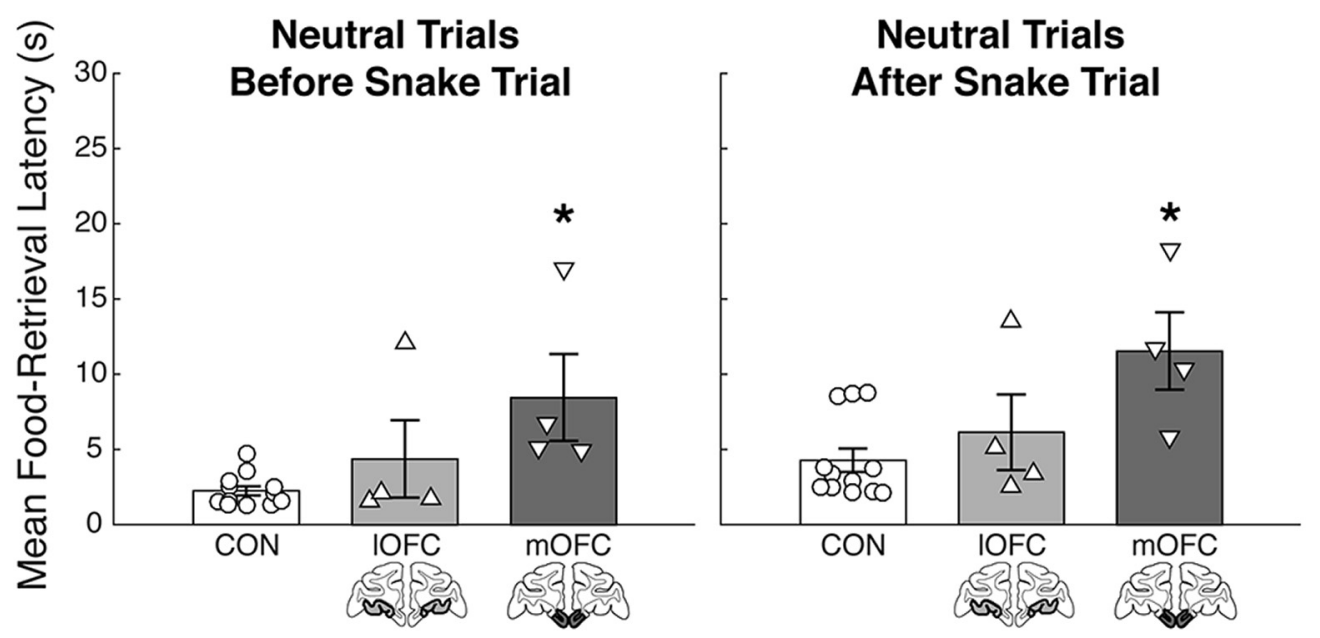

Figure 3. Average food-retrieval latencies collapsed across all five sessions plotted for each group for all neutral object trials preceding the presentation of the fake rubber snake (left) and all neutral object trials following the presentation of the fake rubber snake (right). Labeling conventions as in Figure 2 . Error bar equals SEM; ${ }^{*} p<0.05$ for a comparison of each subtotal $0 \mathrm{FC}$ lesion group to the control group.

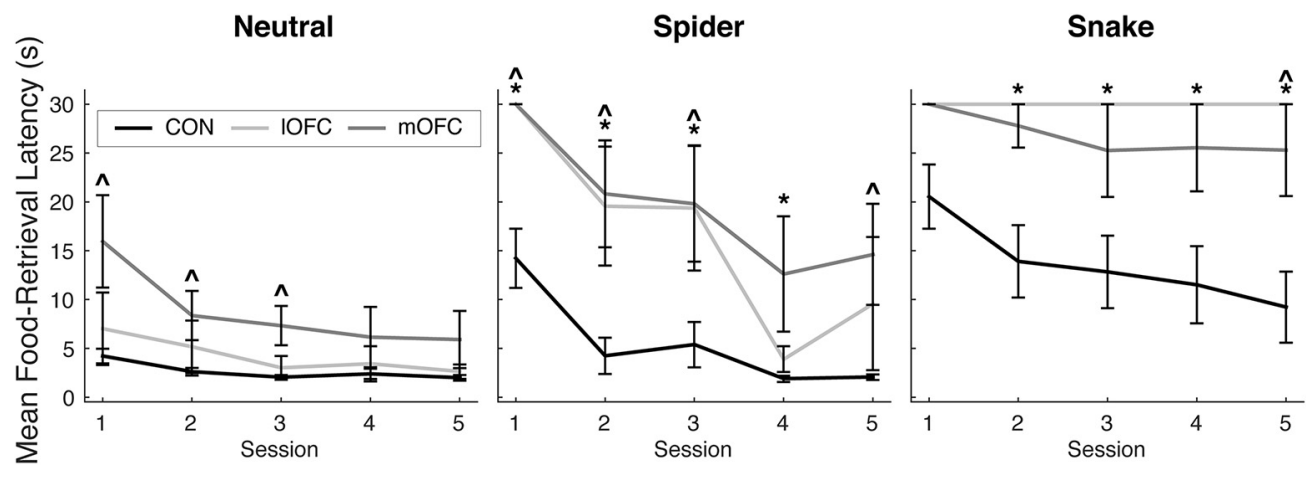

Figure 4. Average food-retrieval latencies plotted for each session for each group separated by trial type (neutral objects, spider, snake). Labeling conventions as in Figure 2. Error bar equals SEM; ${ }^{*} p<0.05$ for a comparison of IOFC group to the control group; $\wedge p<0.05$ for a comparison of the mOFC group to the control group.

significant effect of group for the trials with neutral objects both immediately before $\left(\chi^{2}=8.01, p=0.02\right)$ and immediately after $\left(\chi^{2}=7.09, p=0.03\right)$ the snake trial (Fig. 3). Post hoc tests revealed that this effect was carried by monkeys with mOFC lesions. Relative to controls, this group showed significantly longer foodretrieval latencies for the neutral trials both before $(U=0, p=$ $0.001)$ and after $(U=3, p=0.008)$ the snake trial. There was no significant difference between the lOFC group and controls (before: $U=19, p=0.60$; after: $U=15, p=0.32$ ) or between the operated groups (before: $U=3, p=0.20$; after: $U=3, p=0.20$ ). Lesions of the $\mathrm{mOFC}$, therefore, seemed to have a pervasive effect on retrieval latencies on all neutral trials, and the monkeys' behavior could not simply be explained by carryover effects from snake trials.

We next assessed how retrieval latencies changed over the course of the five test sessions conducted on separate days. Figure 4 shows the mean food-retrieval latencies plotted across all five sessions for each stimulus type. Within-group analyses showed that, as expected, the control group habituated to the threatening stimuli, as evidenced by shorter food-retrieval latencies in the fifth session compared to the first session in the presence of the $\operatorname{spider}(V=78, p=0.0005)$ and the snake $(V=44, p=0.01)$. By contrast, neither operated group habituated to the presence of the spider (lOFC: $V=6, p=0.18$; mOFC: $V=10, p=0.13$ ) or the snake (lOFC: $V=10, p=1.00$; mOFC: $V=10, p=1.00$ ). Between-group analyses showed that, over repeated exposures, the mOFC and lOFC lesion groups continued to show significantly longer food-retrieval latencies in the presence of the snake compared with controls (by the fifth session, lOFC: $U=6, p=$ 0.03; mOFC: $U=7.5, p=0.05)$. For the trials with neutral objects, the mOFC group continued to show longer foodretrieval latencies by the fifth session compared with the controls ( $U=8, p=0.058$, approaching significance). For each category, the operated groups did not differ from each other by the fifth session (spider: $U=4, p=0.34$; snake: $U=10, p=0.45$; neutral: $U=4, p=0.34$ ). In summary, both the lOFC and the mOFC lesion groups exhibited longer latencies to retrieve food in the presence of the threatening stimuli. Notably, the heightened defensive responses were manifest as a failure to habituate to the snake and spider stimuli over repeated exposures. Because longer food-retrieval latencies could be products of other behaviors (e.g., indifference/inattentiveness, overall slowness, etc.), we also assessed the monkeys' whole-body behavioral responses to the stimuli.

\section{Behavioral responses}

To better characterize how subtotal lesions of the OFC affect behavioral responses to threatening stimuli, we conducted ethological assessments of each monkey's behavior. Table 2 provides a description of all the behaviors scored for the approach and defense behavioral categories. There was good interobserver reliability $(r=0.92 ; p=0.001)$. We investigated the proportion of 


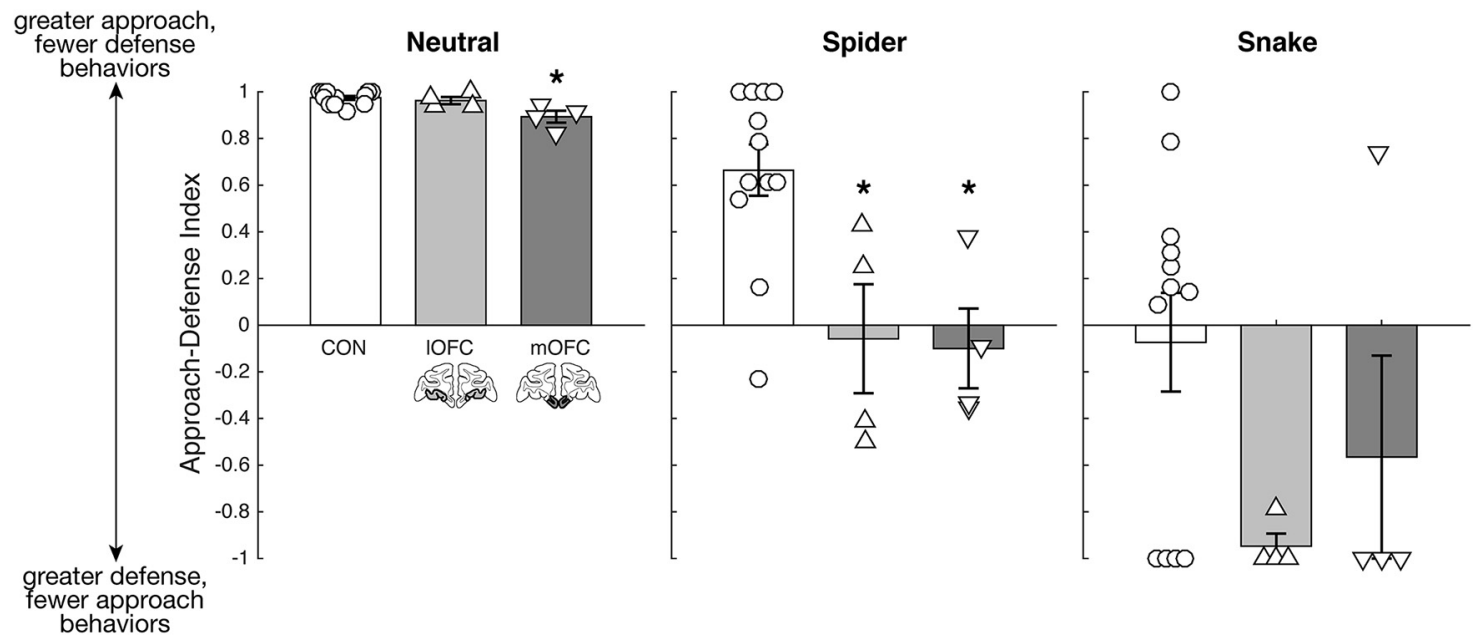

Figure 5. ADI plotted for each group, separated by trial type (neutral stimuli, spider, snake). Labeling conventions as in Figure 2. Error bar equals SEM; ${ }^{*} p<0.05$ for a comparison of each subtotal OFC lesion group to the control group.

Table 3. Summary statistics for approach and defense behaviors

\begin{tabular}{|c|c|c|c|c|c|c|c|c|c|c|}
\hline \multirow[b]{2}{*}{ Condition type } & \multirow[b]{2}{*}{ Behavioral category } & \multicolumn{2}{|c|}{$\operatorname{CON}^{a}(n=12)$} & \multicolumn{2}{|c|}{$\operatorname{IOFC}^{b}(n=4)$} & \multicolumn{2}{|c|}{$\mathrm{mOFC}^{\mathrm{c}}(n=4)$} & \multicolumn{3}{|l|}{$p$ values } \\
\hline & & Mean & SEM & Mean & SEM & Mean & SEM & IOFC vs CON & mOFC vs CON & IOFC vs m0FC \\
\hline \multirow[t]{2}{*}{ Neutral } & Approach & 0.42 & 0.02 & 0.41 & 0.04 & 0.33 & 0.02 & 0.76 & $0.01^{d}$ & 0.20 \\
\hline & Defense & 0.01 & 0.00 & 0.01 & 0.00 & 0.02 & 0.01 & 0.49 & $0.01^{d}$ & 0.14 \\
\hline \multirow[t]{2}{*}{ Spider } & Approach & 0.41 & 0.05 & 0.20 & 0.05 & 0.18 & 0.03 & $0.01^{d}$ & $0.01^{d}$ & 0.88 \\
\hline & Defense & 0.08 & 0.03 & 0.24 & 0.08 & 0.24 & 0.06 & $0.03^{d}$ & $0.02^{d}$ & 1.00 \\
\hline \multirow[t]{2}{*}{ Snake } & Approach & 0.23 & 0.05 & 0.02 & 0.02 & 0.07 & 0.07 & 0.08 & 0.11 & 1.00 \\
\hline & Defense & 0.23 & 0.05 & 0.36 & 0.07 & 0.38 & 0.14 & 0.16 & 0.27 & 1.00 \\
\hline
\end{tabular}

Approach and defense behavioral data for each group and each stimulus type collapsed across all five sessions.

${ }^{a}$ Unoperated control monkeys.

${ }^{b}$ Monkeys with injections of ibotenic acid targeting areas 11 and 13.

'Monkeys with injections of ibotenic acid targeting area 14.

${ }^{d} p<0.05$.

trials that monkeys engaged in approach and defense behaviors and calculated an ADI to characterize behavioral responses for each stimulus category (Fig. 5). Overall, a positive ADI represents greater approach and fewer defense responses, and a negative ADI represents fewer approach and greater defense responses. There was a significant effect of group for the spider trial $\left(\chi^{2}=\right.$ 10.12, $p=0.006)$ but not for the snake trials $\left(\chi^{2}=3.49, p=\right.$ $0.17)$, though a pattern of reduced ADI scores for the subtotal lesion groups is observed. Compared to the controls, both operated groups had lower ADI scores (lOFC: $U=44, p=0.02$; mOFC: $U=45, p=0.01)$ for the spider trials. The operated groups did not differ from each other (spider: $U=7, p=0.89$; snake: $U=7.5, p=1.00)$. Consistent with the food-retrieval latency data, a main effect of group $\left(\chi^{2}=8.01, p=0.02\right)$ and subsequent between-group analyses revealed that the mOFC group had a significantly lower ADI compared with controls ( $U=47, p=0.006$ ), but not compared to the lOFC group $(U=$ $14, p=0.11$ ), in the presence of the neutral stimuli. The lOFC group and controls did not differ from each other $(U=31.5, p=$ $0.38)$.

In sum, relative to controls and consistent with the foodretrieval latency data, both $1 \mathrm{OFC}$ and mOFC lesion groups showed fewer approach and greater defense responses in the presence of threatening stimuli (Table 3). The mOFC group also showed fewer approach and greater defense responses in the presence of the neutral stimuli. Notably, the two operated groups differed in their expression of defensive behaviors. Whereas the
lOFC group withdrew or moved away from the spider more than controls $(U=21, p=0.01)$, the mOFC group displayed more instances of freezing $(U=7.5, p=0.01)$, eye and head aversion $(U=7.5, p=0.04)$, and piloerection $(U=12, p=0.02$; Table 4$)$. Whole-body responses throughout the entire stimulus presentation period therefore provided additional evidence that the experimental groups' increased food-retrieval latencies were due to a combination of reduced approach and increased defense responses to the threatening stimuli.

\section{Discussion}

We found that monkeys with selective, excitotoxic lesions of distinct subdivisions of the OFC - either the lOFC (areas 11/13) or the mOFC (area 14)—showed altered patterns of defensive responses. Relative to controls, both operated groups exhibited increased food-retrieval latencies in the presence of the threatening stimuli. Consistent with this finding, both groups also displayed a pattern of increased defense and decreased approach responses. Strikingly, compared with unoperated controls, monkeys with mOFC damage also showed increased food-retrieval latencies in the presence of the neutral objects. This effect in the neutral condition could not be attributed to the lingering influence of exposure to the fake snake on food-retrieval latencies in subsequent trials with neutral objects. Instead, the effect was due to an overall reduction in approach and increase in defense responses in the presence of the neutral stimuli. Together, these findings 
Table 4. Summary statistics for sub-behaviors scored

\begin{tabular}{|c|c|c|c|c|c|c|c|c|c|c|}
\hline \multirow[b]{2}{*}{ Condition type } & \multirow[b]{2}{*}{ Behavior } & \multicolumn{2}{|c|}{$\operatorname{CON}^{a}(n=12)$} & \multicolumn{2}{|c|}{$\operatorname{lOFC}^{b}(n=4)$} & \multicolumn{2}{|c|}{$\operatorname{m0FC}(n=4)$} & \multicolumn{3}{|l|}{$p$ values } \\
\hline & & Mean & SEM & Mean & SEM & Mean & SEM & IOFC vs CON & mOFC vs CON & IOFC vs mOFC \\
\hline \multirow[t]{8}{*}{ Neutral } & Move forward & 0.00 & 0.00 & 0.02 & 0.01 & 0.01 & 0.01 & $0.002^{d}$ & $0.002^{d}$ & 0.44 \\
\hline & Touch & 0.25 & 0.07 & 0.25 & 0.10 & 0.03 & 0.01 & 1.00 & $0.005^{d}$ & $0.03^{d}$ \\
\hline & Take/eat reward & 1.00 & 0.00 & 0.97 & 0.03 & 0.93 & 0.04 & 0.66 & $0.001^{d}$ & $0.03^{d}$ \\
\hline & Freezing & 0.01 & 0.00 & 0.00 & 0.00 & 0.02 & 0.01 & 0.33 & 0.37 & 0.19 \\
\hline & Startle & 0.00 & 0.00 & 0.01 & 0.01 & 0.01 & 0.01 & 0.79 & 0.79 & 1.00 \\
\hline & Eye/Head Aversion & 0.00 & 0.00 & 0.01 & 0.01 & 0.01 & 0.01 & 0.11 & 0.11 & 1.00 \\
\hline & Piloerection & 0.01 & 0.01 & 0.01 & 0.01 & 0.06 & 0.03 & 1.00 & 0.08 & 0.28 \\
\hline & Move away/withdrawal & 0.00 & 0.00 & 0.01 & 0.01 & 0.01 & 0.01 & 0.46 & 0.46 & 1.00 \\
\hline \multirow[t]{8}{*}{ Spider } & Move forward & 0.00 & 0.00 & 0.05 & 0.05 & 0.00 & 0.00 & 0.11 & 1.00 & 0.45 \\
\hline & Touch & 0.30 & 0.12 & 0.00 & 0.00 & 0.00 & 0.00 & 0.16 & 0.16 & 1.00 \\
\hline & Take/eat reward & 0.93 & 0.04 & 0.54 & 0.14 & 0.55 & 0.10 & $0.006^{d}$ & $0.005^{d}$ & 1.00 \\
\hline & Freezing & 0.02 & 0.02 & 0.05 & 0.05 & 0.25 & 0.13 & 0.46 & $0.01^{d}$ & 0.21 \\
\hline & Startle & 0.05 & 0.03 & 0.10 & 0.10 & 0.10 & 0.06 & 0.87 & 0.41 & 0.87 \\
\hline & Eye/Head Aversion & 0.17 & 0.08 & 0.35 & 0.24 & 0.45 & 0.05 & 0.54 & $0.04^{d}$ & 0.54 \\
\hline & Piloerection & 0.00 & 0.00 & 0.00 & 0.00 & 0.20 & 0.12 & 1.00 & $0.02^{d}$ & 0.18 \\
\hline & Move away/withdrawal & 0.17 & 0.07 & 0.68 & 0.14 & 0.20 & 0.12 & $0.01 d$ & 0.74 & $0.05^{d}$ \\
\hline \multirow[t]{8}{*}{ Snake } & Move forward & 0.03 & 0.03 & 0.05 & 0.05 & 0.00 & 0.00 & 0.53 & 0.67 & 0.45 \\
\hline & Touch & 0.02 & 0.02 & 0.00 & 0.00 & 0.00 & 0.00 & 0.67 & 0.67 & 1.00 \\
\hline & Take/eat reward & 0.63 & 0.14 & 0.00 & 0.00 & 0.20 & 0.14 & $0.04^{d}$ & 0.10 & 0.45 \\
\hline & Freezing & 0.15 & 0.09 & 0.20 & 0.12 & 0.20 & 0.14 & 0.53 & 0.62 & 1.00 \\
\hline & Startle & 0.05 & 0.04 & 0.20 & 0.20 & 0.10 & 0.10 & 0.66 & 0.72 & 1.00 \\
\hline & Eye/Head Aversion & 0.42 & 0.11 & 0.51 & 0.23 & 0.65 & 0.17 & 0.71 & 0.30 & 0.88 \\
\hline & Piloerection & 0.13 & 0.05 & 0.10 & 0.06 & 0.50 & 0.21 & 0.95 & 0.09 & 0.18 \\
\hline & Move away/withdrawal & 0.40 & 0.11 & 0.79 & 0.14 & 0.45 & 0.26 & 0.11 & 1.00 & 0.46 \\
\hline
\end{tabular}

Data for each behavior for each group and each stimulus type collapsed across all five sessions.

${ }^{a}$ Unoperated control monkeys.

${ }^{b}$ Monkeys with lesions of the IOFC.

'Monkeys with lesions of the $\mathrm{mOFC}$.

${ }^{d} p<0.05$.

suggest that medial and lateral sectors of the OFC in intact monkeys serve to downregulate defensive responses.

\section{Role of OFC subregions in responding to threat}

Others have tested the effects of subtotal OFC lesions in macaques on tasks involving predator stimuli and obtained equivocal results (Machado et al., 2009; Noonan et al., 2010a). Several differences between these earlier studies and the present one, including variability in how the lesions were created (e.g., via aspiration, which damages cell bodies and underlying fibers of passage, vs. via injection of excitotoxins, which affects cell bodies only) and differences in testing parameters, preclude identification of the precise factors that account for the different outcomes. Whatever the reasons, our results differ from these earlier reports in macaques and are instead more in line with recent work showing that excitotoxic lesions of the anterior lOFC (primarily area 11 ) and the ventrolateral prefrontal cortex (area 12) in marmosets resulted in heightened defensive responses to a toy snake (Shiba et al., 2014).

The independent contributions of frontal cortical regions to regulating defensive responses both in the marmoset study and the present study would appear to contradict the finding that combined damage to the mOFC and lOFC (i.e., excitotoxic lesions of OFC areas 11,13, and 14) produces no alterations in behavioral responses to threatening stimuli (Rudebeck et al., 2013a). However, differences in anatomical connections of the lOFC and mOFC with other brain regions may explain both the differences between effects of the mOFC and lOFC lesions, as well as the difference from the combined lesion, on behavioral responses to threatening stimuli. Another possibility suggested by the pattern of results, one not mutually exclusive with the first, is that each OFC subregion has an inhibitory influence on the other, either directly or indirectly via a downstream target. This inhibitory interaction could explain the counterintuitive finding that removal of either OFC subregion alone facilitates defensive responses to threat, whereas their combined removal has no effect.

While intact controls habituated to the presence of the threatening stimuli following repeated exposures, monkeys with subtotal OFC damage failed to habituate. Our overall pattern of results therefore suggests a critical role for these two distinct subregions in adaptively attenuating defensive behaviors over time or repeated presentations of a threat stimulus in the absence of overt negative outcomes. These findings should be considered within the context of a larger network of regions known to be involved in the expression and regulation of defensive responses, such as the amygdala, periaqueductal gray, and bed nucleus of the stria terminalis (Fox et al., 2010; DesJardin et al., 2013; Forcelli et al., 2016, 2017; LeDoux and Pine, 2016). With OFC damage or dysfunction, the areas in this network that are critically involved in sustaining arousal and/or marshaling defensive responses may not receive the input necessary to downregulate their activity during multiple exposures to the same threat.

\section{Alternative interpretations}

Although we discuss our findings in the context of the OFC playing a direct role in inhibiting responses to threat, an alternative interpretation is that the OFC subregions guide adaptive learning about safety in the absence of experienced aversive outcomes. This interpretation is in line with the observation that the experimental lesion groups failed to habituate to the predator stimuli following repeated presentations, whereas the control group, on average, showed a significant reduction in defensive responses to the stimuli over time. Further support of this interpretation comes from work showing that defensive responses to snake 
stimuli, but not neutral stimuli, can be conditioned through observational learning, indicating that defensive responses to snakes in macaques, although innate, are capable of being modified through experience (Mineka et al., 1984; Cook et al., 1985; Cook and Mineka, 1989). The exact mechanisms by which the macaque OFC might contribute to learning about safety remain unknown. One possibility is that information about the absence of negative outcomes following their expectation, as a prediction error signal of sorts, may be integrated in areas of the brain described above (e.g., amygdala, periaqueductal gray, etc.) to downregulate behavioral and physiological responses to stimuli.

Although all monkeys were maintained on a controlled diet to ensure motivation to obtain food rewards, yet another possibility is that the lesions disrupted the monkeys' abilities to represent food-reward value, and that this biased their behavior toward defensive responses. This may especially apply to the mOFC lesion group, which exhibited longer food-retrieval latencies in the presence of neutral stimuli. Such an interpretation is consistent with physiological studies in macaques showing that neuronal activity in the lOFC (Wallis and Miller, 2003; Padoa-Schioppa and Assad, 2006; Rudebeck et al., 2013b) and mOFC (Bouret and Richmond, 2010; Strait et al., 2014) reflects reward value. Against this idea, our analysis of whole-body responses suggests that the observed increase in food-retrieval latencies is independent of reward valuation. Monkeys with mOFC damage showed increased defense behaviors to neutral stimuli, specifically freezing in the first session (mOFC vs controls, $U=42, p=0.03$; mOFC vs lOFC, $U=15, p=0.05$ ) and an overall trend toward increased piloerection ( $\mathrm{mOFC}$ vs controls, $U=11, p=0.08$ ), which is difficult to explain on the basis of altered food valuation. Nevertheless, several studies now suggest a role for $\mathrm{mOFC}$ and adjacent regions of the medial frontal cortex in integrating value signals (Bouret and Richmond, 2010; Noonan et al., 2010b; Rudebeck and Murray, 2011; Strait et al., 2014; Howard and Kahnt, 2017; Suzuki et al., 2017), suggesting a role for this area in biasing action selection. Therefore, although an explanation of heightened defensive responses in terms of reduced sensitivity to food value seems unlikely, we cannot rule this possibility out entirely. Future studies will be necessary to isolate the effects of perceived threat from food-reward valuation.

\section{Contribution of OFC subregions to anxiety disorders}

Our data inform findings in humans with anxiety disorders. Human neuroimaging studies have pointed to functional specializations of prefrontal structures in mediating the relationship between emotional reactivity and symptoms of different anxiety disorders (Etkin and Wager, 2007). Based on our pattern of results, it is plausible that dysfunction of specific subdivisions of the OFC may underlie the etiology and symptomatology of different types of anxiety disorders. Generalized defense and hypervigilance consequent to $\mathrm{mOFC}$ damage present similarly to symptoms seen in GAD (Newman et al., 2013) or panic disorder (Lieberman et al., 2016). On the other hand, stimulus-specific hyper-reactivity following lOFC damage appears to be more akin to the symptoms of post-traumatic stress disorder (Lazarov et al., 2019), specific phobia (Hermann et al., 2009), or obsessive-compulsive disorders (Sizino da Victoria et al., 2012; e.g., defensive responses in response to specific "triggering" events or stimuli). Though this interpretation oversimplifies the complexity of anxiety disorder subtypes, it highlights the need for frameworks that propose unique neural substrates underlying distinct symptoms within a common class of disorders (e.g., anxiety).

\section{The OFC and responding to threat: generative versus regulatory role?}

For decades, OFC damage in humans and macaques has been associated with blunted affect (Kleist, 1934; Butter et al., 1970; Blumer and Benson, 1975; Izquierdo et al., 2005; Kalin et al., 2007; Jenkins et al., 2018). The classic blunting or diminished responses to threatening stimuli following OFC damage implies that the OFC is necessary for generating, or expressing, adaptive defensive responses (Hiser and Koenigs, 2018). Blunted affect following OFC damage would therefore appear to contradict models that posit a "top-down" inhibitory role of the prefrontal cortex on limbic and midbrain areas to attenuate defensive responses to perceived threat in animals or fear expression in humans (Likhtik et al., 2005; Thayer and Lane, 2009). The current finding of heightened defensive responses following selective, excitotoxic OFC damage is more consistent with these models. In other words, the results presented here suggest regulatory, as opposed to generative or expressive, roles in defensive behaviors for individual subregions of the OFC and are diametrically opposed to those that follow OFC aspiration lesions in macaques (Butter et al., 1970; Izquierdo et al., 2005; Rudebeck et al., 2006; Kalin et al., 2007). The reasons underlying this difference could be key to understanding the regulation of affect and remain a topic for empirical investigation.

\section{References}

Basile BM, Karaskiewicz CL, Fiuzat EC, Malkova L, Murray EA (2017) MRI overestimates excitotoxic amygdala lesion damage in rhesus monkeys. Front Integr Neurosci 11:12.

Bindi RP, Baldo MVC, Canteras NS (2018) Roles of the anterior basolateral amygdalar nucleus during exposure to a live predator and to a predatorassociated context. Behav Brain Res 342:51-56.

Blumer D, Benson DF (1975) Personality changes with frontal and temporal lobe lesions. In: Psychiatric aspects of neurological diseases (Blumer D, Benson DF, eds), pp 151-170. New York: Grune \& Stratton.

Bouret S, Richmond BJ (2010) Ventromedial and orbital prefrontal neurons differentially encode internally and externally driven motivational values in monkeys. J Neurosci 30:8591-8601.

Butter CM, Snyder DR, McDonald JA (1970) Effects of orbital frontal lesions on aversive and aggressive behaviors in rhesus monkeys. J Comp Physiol Psychol 72:132-144.

Carmichael ST, Price JL (1994) Architectonic subdivision of the orbital and medial prefrontal cortex in the macaque monkey. J Comp Neurol 346: $366-402$.

Carmichael ST, Price JL (1995a) Limbic connections of the orbital and medial prefrontal cortex in macaque monkeys. J Comp Neurol 363:615-641.

Carmichael ST, Price JL (1995b) Sensory and premotor connections of the orbital and medial prefrontal cortex of macaque monkeys. J Comp Neurol 363:642-664.

Chudasama Y, Wright KS, Murray EA (2008) Hippocampal lesions in rhesus monkeys disrupt emotional responses but not reinforcer devaluation effects. Biol Psychiatry 63:1084-1091.

Chudasama Y, Izquierdo A, Murray EA (2009) Distinct contributions of the amygdala and hippocampus to fear expression. Eur J Neurosci 30:23272337.

Cook M, Mineka S (1989) Observational conditioning of fear to fearrelevant versus fear-irrelevant stimuli in rhesus monkeys. J Abnorm Psychol 98:448-459.

Cook M, Mineka S, Wolkenstein B, Laitsch K (1985) Observational conditioning of snake fear in unrelated rhesus monkeys. J Abnorm Psychol 94:591-610.

DesJardin JT, Holmes AL, Forcelli PA, Cole CE, Gale JT, Wellman LL, Gale K, Malkova L (2013) Defense-like behaviors evoked by pharmacological disinhibition of the superior colliculus in the primate. J Neurosci 33:150 155.

Etkin A, Wager TD (2007) Functional neuroimaging of anxiety: a metaanalysis of emotional processing in PTSD, social anxiety disorder, and specific phobia. Am J Psychiatry 164:1476-1488.

Forcelli PA, DesJardin JT, West EA, Holmes AL, Elorette C, Wellman LL, 
Malkova L (2016) Amygdala selectively modulates defensive responses evoked from the superior colliculus in non-human primates. Soc Cogn Affect Neurosci 11:2009-2019.

Forcelli PA, Waguespack HF, Malkova L (2017) Defensive vocalizations and motor asymmetry triggered by disinhibition of the periaqueductal gray in non-human primates. Front Neurosci 11:163.

Fox AS, Shelton SE, Oakes TR, Converse AK, Davidson RJ, Kalin NH (2010) Orbitofrontal cortex lesions alter anxiety-related activity in the primate bed nucleus of stria terminalis. J Neurosci 30:7023-7027.

Greenberg T, Carlson JM, Cha J, Hajcak G, Mujica-Parodi LR (2013) Ventromedial prefrontal cortex reactivity is altered in generalized anxiety disorder during fear generalization. Depress Anxiety 30:242-250.

Hermann A, Schäfer A, Walter B, Stark R, Vaitl D, Schienle A (2009) Emotion regulation in spider phobia: role of the medial prefrontal cortex. Soc Cogn Affect Neurosci 4:257-267.

Hiser J, Koenigs M (2018) The multifaceted role of the ventromedial prefrontal cortex in emotion, decision making, social cognition, and psychopathology. Biol Psychiatry 83:638-647.

Howard JD, Kahnt T (2017) Identity-specific reward representations in orbitofrontal cortex are modulated by selective devaluation. J Neurosci $37: 2627-2638$.

Izquierdo A, Murray EA (2004) Combined unilateral lesions of the amygdala and orbital prefrontal cortex impair affective processing in rhesus monkeys. J Neurophysiol 91:2023-2039.

Izquierdo A, Suda RK, Murray EA (2005) Comparison of the effects of bilateral orbital prefrontal cortex lesions and amygdala lesions on emotional responses in rhesus monkeys. J Neurosci 25:8534-8542.

Jenkins LM, Andrewes DG, Nicholas CL, Drummond KJ, Moffat BA, Phal PM, Desmond P (2018) Emotional reactivity following surgery to the prefrontal cortex. J Neuropsychol 12:120-141.

Kalin NH, Shelton SE, Davidson RJ (2004) The role of the central nucleus of the amygdala in mediating fear and anxiety in the primate. J Neurosci 24:5506-5515.

Kalin NH, Shelton SE, Davidson RJ (2007) Role of the primate orbitofrontal cortex in mediating anxious temperament. Biol Psychiatry 62:1134-1139.

Kleist K (1934) Kriegverletzungen des Gehirns in ihrer Bedeutung für Hirnlokalisation und Hirnpathologie. In: Handbuch der ärztlichen Erfahrungen im Weltkriege, Vol IV (von Schjerning O, ed), pp 343-1408. Leipzig, Germany: Barth.

Lazarov A, Suarez-Jimenez B, Tamman A, Falzon L, Zhu X, Edmondson DE, Neria Y (2019) Attention to threat in posttraumatic stress disorder as indexed by eye-tracking indices: a systematic review. Psychol Med 49:705-726.

LeDoux JE, Pine DS (2016) Using neuroscience to help understand fear and anxiety: a two-system framework. Am J Psychiatry 173:1083-1093.

Lieberman L, Gorka SM, Sarapas C, Shankman SA (2016) Cognitive flexibility mediates the relation between intolerance of uncertainty and safety signal responding in those with panic disorder. Cogn Emot 30:1495-1503.

Likhtik E, Pelletier JG, Paz R, Paré D (2005) Prefrontal control of the amygdala. J Neurosci 25:7429-7437.

Machado CJ, Bachevalier J (2008) Behavioral and hormonal reactivity to threat: effects of selective amygdala, hippocampal or orbital frontal lesions in monkeys. Psychoneuroendocrinology 33:926-941.

Machado CJ, Kazama AM, Bachevalier J (2009) Impact of amygdala, orbital frontal, or hippocampal lesions on threat avoidance and emotional reactivity in nonhuman primates. Emotion 9:147-163.

Málková L, Lex CK, Mishkin M, Saunders RC (2001) MRI-based evaluation of locus and extent of neurotoxic lesions in monkeys. Hippocampus 11:361-370.

Martinez RC, Carvalho-Netto EF, Ribeiro-Barbosa ER, Baldo MV, Canteras NS (2011) Amygdalar roles during exposure to a live predator and to a predator-associated context. Neuroscience 172:314-328.

Meunier M, Bachevalier J, Murray EA, Málková L, Mishkin M (1999) Effects of aspiration versus neurotoxic lesions of the amygdala on emotional responses in monkeys. Eur J Neurosci 11:4403-4418.

Mineka S, Davidson M, Cook M, Keir R (1984) Observational conditioning of snake fear in rhesus monkeys. J Abnorm Psychol 93:355-372.

Mogg K, Bradley BP (2018) Anxiety and threat-related attention: cognitivemotivational framework and treatment. Trends Cogn Sci 22:225-240.

Motta SC, Carobrez AP, Canteras NS (2017) The periaqueductal gray and primal emotional processing critical to influence complex defensive re- sponses, fear learning and reward seeking. Neurosci Biobehav Rev 76:39-47.

Murray EA, Rudebeck PH (2018) Specializations for reward-guided decision-making in the primate ventral prefrontal cortex. Nat Rev Neurosci 19:404-417.

Murray EA, Moylan EJ, Saleem KS, Basile BM, Turchi J (2015) Specialized areas for value updating and goal selection in the primate orbitofrontal cortex. Elife 4:e11695.

Nelson EE, Shelton SE, Kalin NH (2003) Individual differences in the responses of naive rhesus monkeys to snakes. Emotion 3:3-11.

Newman MG, Llera SJ, Erickson TM, Przeworski A, Castonguay LG (2013) Worry and generalized anxiety disorder: a review and theoretical synthesis of evidence on nature, etiology, mechanisms, and treatment. Annu Rev Clin Psychol 9:275-297.

Noonan MP, Sallet J, Rudebeck PH, Buckley MJ, Rushworth MF (2010a) Does the medial orbitofrontal cortex have a role in social valuation? Eur J Neurosci 31:2341-2351.

Noonan MP, Walton ME, Behrens TE, Sallet J, Buckley MJ, Rushworth MF (2010b) Separate value comparison and learning mechanisms in macaque medial and lateral orbitofrontal cortex. Proc Natl Acad Sci U S A 107:20547-20552.

Padoa-Schioppa C, Assad JA (2006) Neurons in the orbitofrontal cortex encode economic value. Nature 441:223-226.

Patrick F, Kempton MJ, Marwood L, Williams SCR, Young AH, Perkins AM (2019) Brain activation during human defensive behaviour: a systematic review and preliminary meta-analysis. Neurosci Biobehav Rev 98:71-84.

Price JL (2005) Free will versus survival: brain systems that underlie intrinsic constraints on behavior. J Comp Neurol 493:132-139.

Rao VR, Sellers KK, Wallace DL, Lee MB, Bijanzadeh M, Sani OG, Yang Y, Shanechi MM, Dawes HE, Chang EF (2018) Direct electrical stimulation of lateral orbitofrontal cortex acutely improves mood in individuals with symptoms of depression. Curr Biol 28:3893-3902.e4.

Rudebeck PH, Murray EA (2011) Dissociable effects of subtotal lesions within the macaque orbital prefrontal cortex on reward-guided behavior. J Neurosci 31:10569-10578.

Rudebeck PH, Buckley MJ, Walton ME, Rushworth MF (2006) A role for the macaque anterior cingulate gyrus in social valuation. Science 313: $1310-1312$.

Rudebeck PH, Saunders RC, Prescott AT, Chau LS, Murray EA (2013a) Prefrontal mechanisms of behavioral flexibility, emotion regulation and value updating. Nat Neurosci 16:1140-1145.

Rudebeck PH, Mitz AR, Chacko RV, Murray EA (2013b) Effects of amygdala lesions on reward-value coding in orbital and medial prefrontal cortex. Neuron 80:1519-1531.

Schienle A, Schäfer A, Hermann A, Rohrmann S, Vaitl D (2007) Symptom provocation and reduction in patients suffering from spider phobia: an fMRI study on exposure therapy. Eur Arch Psychiatry Clin Neurosci 257:486-493.

Shiba Y, Kim C, Santangelo AM, Roberts AC (2014) Lesions of either anterior orbitofrontal cortex or ventrolateral prefrontal cortex in marmoset monkeys heighten innate fear and attenuate active coping behaviors to predator threat. Front Syst Neurosci 8:250.

Shiba Y, Santangelo AM, Roberts AC (2016) Beyond the medial regions of prefrontal cortex in the regulation of fear and anxiety. Front Syst Neurosci 10:12.

Sizino da Victoria M, Nascimento AL, Fontenelle LF (2012) Symptomspecific attentional bias to threatening stimuli in obsessive-compulsive disorder. Compr Psychiatry 53:783-788.

Strait CE, Blanchard TC, Hayden BY (2014) Reward value comparison via mutual inhibition in ventromedial prefrontal cortex. Neuron 82:13571366.

Suzuki S, Cross L, O’Doherty JP (2017) Elucidating the underlying components of food valuation in the human orbitofrontal cortex. Nat Neurosci 20:1780-1786

Thayer JF, Lane RD (2009) Claude Bernard and the heart-brain connection: further elaboration of a model of neurovisceral integration. Neurosci Biobehav Rev 33:81-88.

Walker AE (1940) A cytoarchitectural study of the prefrontal area of the macaque monkey. J Comp Neurol 73:59-86.

Wallis JD, Miller EK (2003) Neuronal activity in primate dorsolateral and orbital prefrontal cortex during performance of a reward preference task. Eur J Neurosci 18:2069-2081. 